\title{
Freeze-drying cycle optimization for the rapid preservation of protein- loaded liposomal formulations
}

\author{
Maryam T Hussain ${ }^{\mathrm{a}}$, Neil Forbes ${ }^{\mathrm{a}}$, Yvonne Perrie $^{\mathrm{a}}$, Kiran P Malik ${ }^{\mathrm{b}}$, Chinwe Duru ${ }^{\mathrm{b}}$, Paul Matejtschuk $\mathrm{b}^{\mathrm{b}^{*}}$ \\ aStrathclyde institute of Pharmacy and Biomedical science, University of Strathclyde, Glasgow, G4 ORE, \\ Scotland; \\ ${ }^{\text {b }}$ Standardisation Science, National Institute for Biological Standards \& Control (NIBSC), Blanche Lane, \\ South Mimms, Potters Bar, United Kingdom EN6 3QG.
}

*Corresponding author

\begin{abstract}
Technology such as the use of microfluidics to generate liposomes has been well researched, yet the stabilisation of liposomal formulations is a major challenge to their greater implementation. To the best of our knowledge, this is the first study investigating the use of 96 well plates to freeze-dry ovalbumin (OVA) loaded neutral (DMPC:Chol and DSPC:Chol), anionic (DSPC:Chol:PS) and cationic (DSPC:Chol:DOTAP) liposomes. Through the use of high throughput screening, a freeze drying cycle was optimised; ramp freezing from from $4^{\circ} \mathrm{C}$ to $-45^{\circ} \mathrm{C}$, followed by primary drying at $-30^{\circ} \mathrm{C}$ and secondary drying at $30^{\circ} \mathrm{C}$ under a vacuum of $0.1 \mathrm{mBar}$. These parameters maintained liposome physicochemical properties, with the liposomes remaining below $100 \mathrm{~nm}$ and were homogenous (polydispersity index of less than 0.2 post rehydration). Minimal leakage of the OVA protein was observed, with almost $100 \%$ OVA remaining encapsulated post rehydration of the formulations. Here we have identified a simple method that allows for the rapid screening and freeze-drying of a range of liposomal formulations.
\end{abstract}


Graphical Abstract

Liposomes
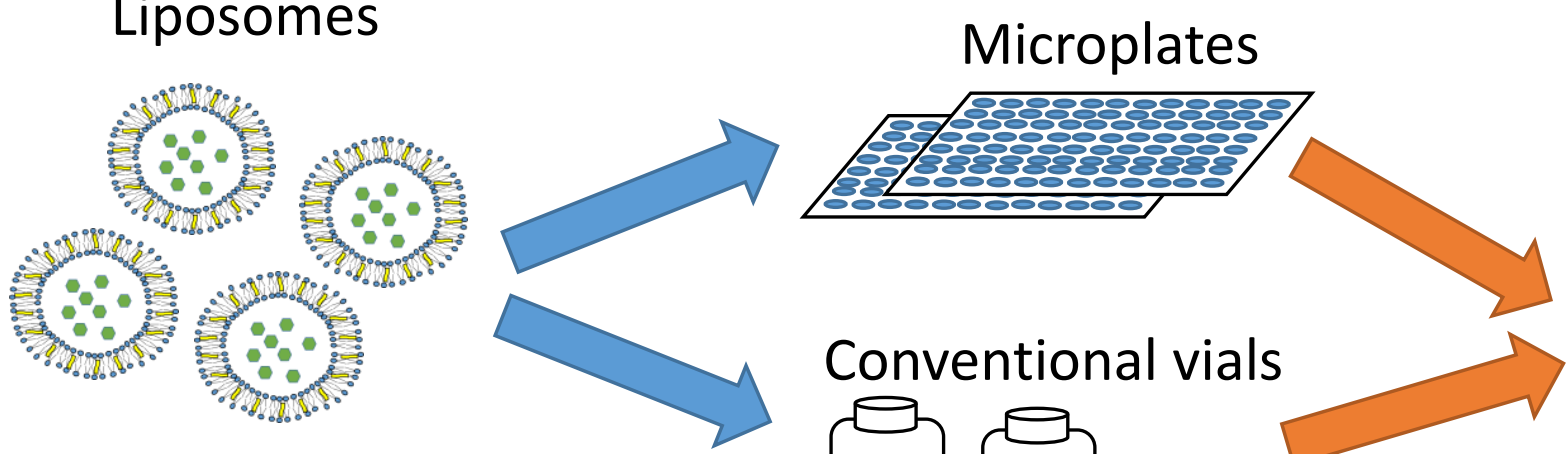

Conventional vials 


\section{Introduction}

Liposomal delivery systems are increasingly used in the pharmaceutical industry due to their versatility. Recent advances in manufacturing using chip technology such as microfluidics, has the potential to overcome bottlenecks experienced, thus liposome colloidal systems are increasingly researched as delivery systems and vaccine adjuvants $[1,2]$. Whilst colloidal systems are good delivery systems, due to the complex nature of the formulations, maintaining stability of the formulations is often difficult. Liposomes are prone to oxidation or hydrolysis caused by chemical [3] and physical degradation [4], drug leakage, formation of aggregates, and fusion. All these changes can alter the formulation properties consequently impacting the in-vivo bio-distribution and pharmacokinetic properties of the drug formulation $[2,5,6]$. The liposomal composition itself is well studied and known to influence stability; lipids (use of saturated lipids and high transition lipids), lipid charge, presence of protein, the amount of cholesterol and cryoprotectant concentrations are well known to impact stability. Although marketed liposomal formulations in liquid form do exist, such as Doxil $^{\circledR}$ and Abelcet ${ }^{\circledR}$ formulations which can be stored for around 24 to 36 months [5, 7-10] it is very expensive to maintain a cold chain when exporting worldwide. In cases where formulation changes and ideal storage conditions (inert atmosphere of $-20^{\circ} \mathrm{C}$ ) are not sufficient at maintaining stability, other approaches such as freeze-drying liposomal formulations can be considered.

Freeze drying is the most popular and studied technique for the stabilisation of liposomes. In itself, freeze drying is a complex process and consists of three main stages; freezing (of liposomecryoprotectant mixtures), primary drying (where sublimation occurs) and secondary drying (to remove residual moisture content). The correct selection of cryoprotectant, excipients, and process parameters (like freezing and drying temperatures) are required to maintain liposome bilayer integrity [11]. The use of powerful freeze drying analytical equipment and better understanding of the process has revolutionised the process scale lyophilisation of protein therapeutics. For instance, Grant and colleagues used a design of experiments (DoE) approach to determine the relationship between variable factors and the outcome of the freeze drying process $[12,13]$. Using a micro-scale model (96 well plates) along with DoE they were able to rapidly optimize, screen and identify factors such as excipients impacting the freeze drying of the protein lactose dehydrogenase [13].

Despite a range of protocols existing for the freeze drying of liposomes [14, 15], reproducibility and high-throughput screening of freeze dried liposomal formulations is challenging. To the best of our knowledge, rapid screening of liposomes using a scale down approach (microplates) has not been investigated. Taking into consideration known factors effecting liposomal stability, this work aims to identify the formulation and process parameters ideal for the freeze drying of empty and protein loaded liposomes (using Ovalbumin (OVA)). Special attention was also paid to the formulation parameters with regards to lipid selection, lipid charge (neutral, cationic and anionic), lipid concentration and the final concentration of the cryoprotectant sucrose as all these factors are known to influence freeze-drying. Given the complexity of the formulations, and the need for rapid throughput, special attention will be paid to identifying parameters using the DoE and relevant control approaches.

\section{Materials and Methods}

\subsection{Materials}

The lipids 1,2-dimyristoyl-sn-glycero-3-phosphocholine (DMPC), 1,2-distearoyl-sn-glycero-3phosphocholine (DSPC), 1,2-dioleoyl-3-trimethylammonium-propane (DOTAP) and L- $\alpha$ - 
phosphatidylserine (Brain PS, Porcine) were all obtained from Avanti Polar Lipids Inc., Alabaster, AL, US. Cholesterol, Ovalbumin (OVA), sucrose, D9777-100ft dialysis tubing cellulose and trifluoroacetic acid was obtained from Sigma Aldrich Company Ltd., Poole, UK. For liposome purification (removal of organic solvent and OVA protein) by Tangential flow filtration (TFF), a modified polyethersulfone (mPES) 750kD MWCO hollow fibre column was purchased from Spectrum Inc., Breda, The Netherlands. A Jupiter column (C18 (300 Å), $5 \mu \mathrm{m}$, dimensions $4.60 \times 150 \mathrm{~mm}$ ) was procured from Phenomenex, Macclesfield, UK. For quantification of protein, HPLC grade methanol and 2-propanol were purchased from Fisher Scientific., Loughborough, England, UK. The water and solvents used were HPLC grade. For freeze dry liposomes, $2 \mathrm{~mL}$ Fiolax clear glass type I vials (35mm x $16 \mathrm{~mm}$ diameter) (Schott VCDIN2R) were used. These and the accompanying closures (13mm halobutyl igloo closures) were obtained from Adelphi Healthcare Packaging, (Haywards Heath, UK). The 96 well plates were obtained from Thermo Scientific Supplies (Hemel Hempstead, UK).

\subsection{Methods}

\subsubsection{Production, purification and characterisation of ovalbumin loaded liposomes using microfluidics}

Lipids and cholesterol were dissolved in methanol at varying concentrations (maintaining a 2:1 wt/wt ratio) and injected into the solvent port of the Precision Nanosystem Benchtop NanoAssembler (Precision Nanosystems, Vancouver, Canada). OVA was solubilised in phosphate buffered saline (PBS) $(10 \mathrm{mM}, \mathrm{pH} 7.3)$ at varying concentrations $(0-0.25 \mathrm{mg} / \mathrm{mL})$ and injected into the second inlet. The liposomal formulations were produced at a 3:1 flow rate ratio (FRR) (the ratio between the organic and aqueous phase) and at a speed of $15 \mathrm{~mL} / \mathrm{min}$ (referred to as the total flow rate (TFR)). The positively charged DSPC:Chol:DOTAP liposomes (10:5:4 wt/wt) were produced at a 1:1 FRR and 15 $\mathrm{mL} / \mathrm{min}$ TFR. Liposomal formulations were purified to remove residual organic solvent and nonincorporated OVA by KrosFlo ${ }^{\circledR}$ Research 2i Tangential flow filtration system (TFF) (Spectrum labs, California, USA). Briefly, the samples are flushed with phosphate buffered saline (PBS) $(10 \mathrm{mM}, \mathrm{pH}=$ 7.3) at a speed of $27 \mathrm{~mL} / \mathrm{min}$ to remove solvent and unentrapped protein. Post purification, the formulations were characterised using dynamic light scattering (DLS) (Malvern Zetasizer NanoZS, Malvern Instruments, Malvern, UK). The Z-average and polydispersity index (PDI) were obtained by diluting liposomes to PBS (1:10) to measure the size and PDI.

\subsubsection{Quantification of ovalbumin loading in liposomal formulations}

For the quantification of OVA protein in liposomal formulations, high performance liquid chromatography (HPLC) coupled with evaporative light scattering detector (ELSD) was used. A previously published method that directly measured encapsulated OVA was followed ${ }^{17}$. Briefly, following manufacture, purified liposomes (with non-entrapped OVA and solvent removed by TFF) were added to a 50/50 v/v ratio of solubilisation solution (PBS / 2-Propanol 50/50 v/v) and vortexed. A similar procedure was followed post rehydration of freeze-dried samples. The formulations were run through the TFF to remove OVA that may have leaked as a result of freeze drying and reconstitution. The liposomal formulations were then solubilised using the technique described above, and the encapsulated OVA was quantified directly using HPLC-ELSD (at a flow rate of $1 \mathrm{~mL} / \mathrm{min}$, with the OVA peak appearing at 11.8 minutes). The amount of encapsulated OVA in liposomes produced by microfluidics, was calculated using the peak area of the sample in relation to an established calibration curve. The encapsulation efficiency (\%) was calculated by dividing the amount of encapsulated OVA by the total amount of OVA protein. 


\subsubsection{Freeze dried microscopy}

The freeze dried microscope (FDM) was used to help determine the parameters for the freeze dry cycle. The FDM allows an estimation of the freezing, collapse and melt temperature of liposomecryoprotectant mixtures (at a 1:1 ratio (v/v). This was achieved by using a Linkam FDCS 196 cryostage mounted on a BX51 Olympus optical microscope connected to a Linkam control unit (TMS 94, VC 94, LNP, Linkam Scientific Instruments Ltd, Tadworth, Surrey, UK) for visualisation. The samples were frozen using liquid nitrogen at a rate of $10^{\circ} \mathrm{C} / \mathrm{min}$ until $-50^{\circ} \mathrm{C}$ was reached, after which the temperature was held for 2 minutes before drying was started by applying a vacuum. The samples were freeze dried under $0.1 \mathrm{mBar}$ vacuum. To establish the collapse and melt of the samples, the temperature was then ramped up to $20^{\circ} \mathrm{C}$ at a controlled rate. Images were taken every 20 seconds for the duration of the cycle.

\subsubsection{Modulated Differential scanning calorimetry (MDSC)}

Modulated differential scanning calorimetry (MDSC) was performed using a Q2000 DSC (TA Instruments, Elstree, Hertfordshire, UK). Following loading of the sample (liposome-cryoprotectant mixture containing $7.5 \%$ final sucrose after mixing) and empty reference pan the unit was cooled to $90^{\circ} \mathrm{C}$ at $10^{\circ} \mathrm{C} /$ min marking the end of cycle 1 ; the thermal changes were recorded from this point on. Cycle 2 consisted of holding the sample at isothermal conditions for eight minutes after which the sample was ramped up at $1.5^{\circ} \mathrm{C} / \mathrm{min}$ with modulation at $0.23^{\circ} \mathrm{C} / \mathrm{min}$ until $25^{\circ} \mathrm{C}$ was reached. Three data sets were generated; heat flow, reversing heat flow and non-reversing heat flow. The reversing heat flow (blue line) was used to determine the glass transition values.

\subsubsection{Dynamic mechanical analysis}

The dynamic mechanical analysis technique (DMA Q800, TA Instruments, Elstree, UK) was used to identify the collapse temperature of liposome-cryoprotectant mixtures. Approximately $100 \mu \mathrm{L}$ of liposome-cryoprotectant mixture was loaded evenly onto a filter paper (ThermoFisher product number E1, $89 \times 140 \mathrm{~mm}, \mathrm{Box} / 100$ ) cut to size to fit the large steel sample holder. Once added to the instrument, the sample was held at $-70^{\circ} \mathrm{C}$ for five minutes before the temperature was ramped to $5^{\circ} \mathrm{C}$ at $1^{\circ} \mathrm{C} / \mathrm{min}$, with $1 \mathrm{bar}$ of compressed nitrogen pressure applied. A stress frequency of between 1 and $100 \mathrm{~Hz}$ was applied, and the sample measured.

\subsubsection{Freeze drying cycle}

Freeze drying cycles were investigated for the freeze drying of protein loading liposomal formulations using the Telstar Lyobeta 15 (Telstar Azbil , Terrassa, Spain). Samples to be freeze dried were pipetted into either $2 \mathrm{~mL}$ vials or into 96 well flat bottom plates. The 96 well plates had the skirt of the plates removed to allow direct thermal contact of the bottom of the wells with the shelf. Freezing of the formulations varied; it involved either pre-cooling, ramp freeze or snap freezing of the formulations. The results from the FDM and MDSC results were used to create a freeze drying cycle. Three freeze drying cycles were investigated for the ability to preserve liposomal formulations. The first freeze drying cycle (FDC1) involved pre-cooling the shelf to $-45^{\circ} \mathrm{C}$, after which the primary drying phase involves increasing the shelf temperature from $-45^{\circ} \mathrm{C}$ to $-30^{\circ} \mathrm{C}$ where it was held for 10 hours at 0.1 
mBar vacuum. The secondary drying phase involved increasing the temperature to $20^{\circ} \mathrm{C}$ and holding the temperature for 5 hours. The physicochemical properties of the liposomal formulations post rehydration, showed an increase in liposome size. Based on this, the existing freeze drying cycle 1 was adapted, with changes to the freezing portion of the cycle. Two different freeze drying cycles were investaigted. The samples were either ramp frozen or snap frozen, to determine if this would prevent changes in the size of the liposomal formulations.

The second freeze drying cycle (FDC2) ran using a ramped freeze approach; with the samples frozen from $4^{\circ} \mathrm{C}$ to $-45^{\circ} \mathrm{C}$, over a 90 min period. The samples were held for 180 minutes at $-45^{\circ} \mathrm{C}$ after which a vacuum of $0.1 \mathrm{mBar}$ was applied for 30 minutes. The primary drying involved ramping up the temperature to $-30^{\circ} \mathrm{C}$ (the process taking 30 minutes); the temperature was maintained for a total of 1320 minutes. For the secondary drying phase, the temperature was increased from $-30^{\circ} \mathrm{C}$ to $30^{\circ} \mathrm{C}$ then held for 360 minutes under a vacuum of $0.1 \mathrm{mBar}$.

The third freeze drying cycle (FDC3) involved snap freezing liposome-sucrose mixtures using liquid nitrogen, before loading onto a pre-cooled freeze-drying shelf. The first drying cycle involved ramping the temperature up to $-30 \mathrm{C}$ (in 30 minutes at $0.1 \mathrm{mb}$ ) after which it was held at this temperature for 600 minutes. The secondary drying stage involved a temperature ramp from $-30 \mathrm{C}$ to $30 \mathrm{C}$ then holding this temperature for 360 minutes, whilst maintaining the pressure of $0.1 \mathrm{mBar}$.

\subsubsection{Measuring residual moisture content using TGA}

Thermogravimetric analysis (TGA) was used to measure the moisture content of the freeze dried liposome preparations. A Pyris-1 TGA (Perkin Elmer Thermal Analysis, Seer Green, UK) fitted with the Accupik autosampler and interfaced with evolved gas analysis on a Hidden HPR 20 (Hiden Ltd, Warrington, UK) was used. Samples were panned under low humidity conditions ( $\mathrm{RH}<10 \%)$ in a Captair pyramid dry bag (Cole Parmer, London, UK) continuously flushed with dry nitrogen. The sealed pans were loaded on the Accupick and punctured automatically just prior to loading. Samples were heated at $20^{\circ} \mathrm{C} /$ minute from ambient to $300^{\circ} \mathrm{C}$ [16] and the resultant weight loss profile compared with the carbon dioxide and water profiles from the gas analysis to estimate the water content related to bound water and prior to thermal degradation of the sample [16]. Samples were analysed and the mean weight loss $\mathrm{w} / \mathrm{w} \%$ reported. The system performance was checked using a sodium tartarate standard of known moisture content (Mitsubishi Dry moisture standard, obtained from A1 Envirosciences Ltd, Blyth, UK).

\subsubsection{Data collection and analysis}

The statistical software package, MODDE11 (Umetrics, Sartorius-Stedim Biotech, Sweden) was used to identify the ideal critical process parameters. Utilizing predictive modelling, a set of primary conditions (liposomes concentration, sucrose concentration and encapsulation of OVA) was investigated to identify the ideal CPP. A full $2^{3}$ factorial study was conducted at a $95 \%$ confidence interval. The outputs of the experiments took into consideration changes in size, PDI and encapsulation efficiency when predicting the best freeze drying parameters. The critical process parameters were tested using FDC1 parameter (described in section 2.2.6). Once the samples had been rehydrated and analysed, the liposomal formulations had shown an increase in size and so alternative FDC parameters were tested. 
For all other experiments, ANOVA and T- tests were used to assess statistical significance ( $p$ value of less than 0.05 ), with a post Tukey's ad-hoc test performed.

\section{Results}

\subsection{Establishing the physicochemical properties of liposomal formulations before freeze drying}

The OVA loaded liposomal formulations were produced using microfluidics, at a 3:1 flow rate ratio (FRR) and $15 \mathrm{~mL} / \mathrm{min}$ as these parameters are optimal for the maximum encapsulation of protein [17]. The DMPC $\left(\mathrm{Tm}=23^{\circ} \mathrm{C}\right)$ and DSPC $\left(\mathrm{Tm}=55^{\circ} \mathrm{C}\right)$ lipids were selected as examples of low and high transition temperature lipids. Prior to testing freeze drying conditions, the liposomal formulations were characterised in order to identify any changes post freeze drying. The results from Table 1 show the physicochemical properties of DMPC:Chol and DSPC:Chol liposome formulations produced with and without OVA. Manufacture of liposomes by microfluidics produced liposomes below $100 \mathrm{~nm}$ in size, with a PDI of below 0.2 and a high encapsulation efficiency (above 30\%) [17]. We have shown in a previous research paper that manufacturing of protein loaded liposomes by microfluidics does not cause denaturation [17]. Circular dichroism results showed the protein structure of OVA was maintained when producing OVA loaded liposomes by microfluidics despite the presence of solvent [17], and so this was not investigated in this research paper. Whilst producing high quality liposomes is possible using microfluidics, maintaining liposome stability is important, with freeze drying a viable option.

Furthermore, to determine the effect of liposomal membrane charge on particle stability after the freeze drying process, anionic and cationic formulations were produced both with and without the incorporation of OVA. Anionic formulation DSPC:Chol:PS was produced using microfluidics under the same manufacturing parameters as DMPC:Chol and DSPC:Chol, while cationic liposomal formulation DSPC:Chol:DOTAP was produced at a flow rate ratio of $1: 1$ with a $15 \mathrm{~mL} / \mathrm{min}$ TFR. Formulations without the inclusion of OVA resulted in particle sizes of $42 \mathrm{~nm}$ and $64 \mathrm{~nm}$ for DSPC:Chol:PS and DSPC:Chol:DOTAP respectively, with PDI values of 0.15 and below (Table 2). Encapsulation of OVA within the aqueous core of the anionic particles resulted in particle sizes of $145 \mathrm{~nm}$ with PDI below 0.2 , in comparison to the surface adsorption of OVA for the cationic liposomal formulations which may have resulted in particle sizes of $253 \mathrm{~nm}$ (retaining a PDI of 0.2). Anionic formulation DSPC:Chol:PS encapsulated $372 \pm 19.7 \mu \mathrm{g} / \mathrm{mL}$, with an encapsulation efficiency $6.6 \%$ (initial OVA concentration of $7.5 \mathrm{mg} / \mathrm{mL})$. 
Table 1: The physicochemical parameters of DMPC:Chol and DSPC:Chol liposomal formulations produced using microfluidics at a 3:1 flow rate ratio (FRR) and $15 \mathrm{~mL} / \mathrm{min}$ total flow rate (TFR). The lipid concentration was varied alongside the presence of the protein OVA $(0.125 \mathrm{or} 0.25 \mathrm{mg} / \mathrm{mL})$ to determine the impact on liposomal physicochemical properties. The size (d. $\mathrm{nm}$ ) and polydispersity index was measured using dynamic light scattering with the amount of entrapped OVA and encapsulation efficiency (EE \%) determined using HPLC-ELSD.

\begin{tabular}{|c|c|c|c|c|c|c|c|}
\hline FORMULATIONS & Tests & $\begin{array}{l}\text { Lipid } \\
\text { concentration } \\
(\mathrm{mg} / \mathrm{mL})\end{array}$ & $\begin{array}{l}\text { Ovalbumin } \\
(\mathrm{mg} / \mathrm{ml})\end{array}$ & Size (d. nm) & $\begin{array}{l}\text { POLYDISPERSITY } \\
\text { INDEX }\end{array}$ & $\begin{array}{l}\text { ENCAPSULATION } \\
\text { EFFICIENCY (\%) }\end{array}$ & $\begin{array}{l}\text { Amount entrapped } \\
(\mu \mathrm{g} / \mathrm{mL})\end{array}$ \\
\hline \multirow[t]{5}{*}{ DMPC:Chol } & 1 & 4 & 0 & $79 \pm 0.5$ & $0.12 \pm 0.08$ & - & - \\
\hline & 2 & 10 & 0 & $79 \pm 0.5$ & $0.09 \pm 0.017$ & - & - \\
\hline & 3 & 4 & 0.25 & $96 \pm 1.2$ & $0.21 \pm 0.005$ & 37 & 70 \\
\hline & 4 & 10 & 0.25 & $88 \pm 0.2$ & $0.22 \pm 0.012$ & 39 & 74 \\
\hline & 5 & 7 & 0.125 & $91 \pm 0.1$ & $0.25 \pm 0.005$ & 49 & 46 \\
\hline \multirow[t]{5}{*}{ DSPC:Chol } & 6 & 4 & 0 & $46 \pm 0.1$ & $0.15 \pm 0.005$ & - & - \\
\hline & 7 & 10 & 0 & $56 \pm 3.0$ & $0.21 \pm 0.005$ & - & - \\
\hline & 8 & 4 & 0.25 & $65 \pm 3.7$ & $0.27 \pm 0.050$ & 34 & 65 \\
\hline & 9 & 10 & 0.25 & $88 \pm 0.2$ & $0.23 \pm 0.010$ & 36 & 68 \\
\hline & 10 & 7 & 0.125 & $57 \pm 4.0$ & $0.22 \pm 0.007$ & 48 & 45 \\
\hline
\end{tabular}


Table 2: The physicochemical properties of DSPC:Chol:PS and DSPC:Chol:DOTAP liposomes made using microfluidics at a 3:1 flow rate ratio (FRR) and 15 $\mathrm{mL} / \mathrm{min}$ total flow rate (TFR). The formulation were manufactured with or without the protein OVA at different concentrations. Dynamic light scattering was used to determine the size $(\mathrm{nm})$ and polydispersity index. The amount of encapsulated OVA and determined using HPLC-ELSD, with the amount of OVA adsorbed onto the cationic DSPC:Chol:DOTAP not quantified.

\begin{tabular}{|c|c|c|c|c|c|c|}
\hline FORMULATION & $\begin{array}{l}\text { Lipid concentration } \\
(\mathrm{mg} / \mathrm{ml})\end{array}$ & $\begin{array}{l}\text { Ovalbumin } \\
(\mathrm{mg} / \mathrm{ml})\end{array}$ & Size (d. nm) & $\begin{array}{l}\text { POLYDISPERSITY } \\
\text { INDEX }\end{array}$ & $\begin{array}{l}\text { ENCAPSULATION } \\
\text { EFFICIENCY (\%) }\end{array}$ & $\begin{array}{l}\text { Amount entrappec } \\
(\mu \mathrm{g} / \mathrm{mL})\end{array}$ \\
\hline \multirow[t]{2}{*}{ DSPC:Chol:PS } & 10 & 0 & $79 \pm 0.5$ & $0.12 \pm 0.08$ & - & - \\
\hline & 10 & $7.5 \mathrm{mg} / \mathrm{mL}$ & $91 \pm 0.1$ & $0.25 \pm 0.005$ & $6.61 \%$ & 372.6 \\
\hline \multirow[t]{2}{*}{ DSPC:Chol:DOTAP } & 10 & 0 & $46 \pm 0.1$ & $0.15 \pm 0.005$ & - & - \\
\hline & 10 & $1 \mathrm{mg} / \mathrm{mL}$ & $56 \pm 3.0$ & $0.21 \pm 0.005$ & - & - \\
\hline
\end{tabular}




\subsection{Optimising the freeze drying parameters (by identifying the freezing and collapse temperature)}

Due to the nature of the liposomes, which are extremely susceptible to stress caused by changes in pressure and temperature during the freeze drying cycle, the cycle requires a lot of research and refinement. To aid with the freeze drying cycle development, analytical techniques can be used (alone or in combination). Freeze drying microscopy (FDM), modulated differential scanning calorimetry (MDSC) and dynamic mechanical analysis (DMA) were all considered for this.

The FDM was used to predict the ideal freeze drying conditions for liposome-cryoprotectant mixtures. Both neutral and anionic liposomes were mixed with the cryoprotectant sucrose at a $7.5 \%$ final sucrose concentration, at a 1:1 ratio ( $\mathrm{v} / \mathrm{v}$ ) (Table 3). Physical changes of the liposome-sucrose mixture can be observed in real-time for all four liposomal formulations (OVA loaded DSPC:Chol, DMPC:Chol, OVA loaded DMPC:Chol and OVA loaded DSPC:Chol:PS) investigated. The freezing temperature for DMPC:Chol $\left(-23^{\circ} \mathrm{C}\right)$, OVA loaded DMPC:Chol $\left(-19^{\circ} \mathrm{C}\right)$, OVA loaded DSPC:Chol $\left(-19^{\circ} \mathrm{C}\right)$ and OVA loaded DSPC:Chol:PS $\left(-19^{\circ} \mathrm{C}\right)$ are within a tight range irrespective of the charge (Table 3$)$. The collapse temperatures of the liposome-sucrose mixtures are also within a tight range; between -39 and $-33^{\circ} \mathrm{C}$ regardless of lipid type, the lipid concentration, the presence of OVA or the charge of the formulation (Table 3) suggesting the collapse point is largely influenced by the presence of sucrose. The product temperature during primary drying must not exceed the collapse temperatures to ensure maximum freeze drying of the liposome formulations. The changes in liposome-cryoprotectant morphology can be also be observed; with the freezing (Figure 1B), drying (Figure 1C) and collapse (Figure 1D) identifiable.

In addition, liposome-sucrose mixtures were analysed by another popular technique; modulated differential scanning calorimetry (MDSC) to detect thermal changes the formulation experiences when cooled and heated over a range of temperatures. Determining the glass transition temperature $\left(\mathrm{Tg}^{\prime}\right)$ in the frozen state is important, as the primary drying phase must not exceed this in order to avoid collapse and to form an elegant cake. Once again, the three formulations studied were not influenced by the presence of protein, lipid or lipid concentration (Table 1). The $\mathrm{Tg}^{\prime}$ values for OVA loaded DMPC:Chol liposome mixture is $-36.2^{\circ} \mathrm{C},-36.2^{\circ} \mathrm{C}$ for empty DMPC:Chol liposome mixture and a $\mathrm{Tg}^{\prime}$ of $-36.1^{\circ} \mathrm{C}$ for DSPC:Chol formulations. $\mathrm{Tg}^{\prime}$ values from MDSC confirmed that the effect of a negatively charged liposomal membrane did not affect the glass transition of the system, with highly comparable results being obtained from that of the neutral liposomes $\left(-36.2^{\circ} \mathrm{C}\right)$. As with FDM, the cryoprotectant (in this case sucrose) is dominating the $\mathrm{Tg}^{\prime}$ of the formulation- cryoprotectant mixture. The sugars plays a vital role in stabilising the liposome formulations; in the absence of cryoprotectants the lipid head group are in close proximity resulting in increased van der Waal's forces between the lipids resulting in increases $\mathrm{Tg}^{\prime}$. Stability can be improved by adding cryoprotectant which as a result reduces the $\operatorname{Tg}^{\prime}$ values $[18,19]$.

From the MDSC curves, the $\mathrm{Tg}^{\prime}$ values are hard to quantify as the curve is dominated by the melt peak starting from $-20^{\circ} \mathrm{C}$. Although it has been claimed PBS can cause precipitation of the buffer during freeze drying, recent research by [20] has shown the addition of sucrose can prevent this, and so the MDSC curve is dominated by the water melt peak. As a result dynamic mechanical analysis (DMA) was tested as an alternative for the liposome-cryoprotectant mixture. This analytical technique applies stress and strain to the liposome- cryoprotectant mixture to measures changes in morphology, quoted as tan Delta. The values calculated for the OVA loaded DSPC:Chol, DMPC:Chol, OVA loaded DMPC:Chol are between -23 and $-24^{\circ} \mathrm{C}$ (Table 2). For anionic liposomes (OVA loaded DSPC:Chol:PS) a similar trend was observed with DMA, where the tan Delta value is higher than the value obtained through MDSC, 
$-17.4^{\circ} \mathrm{C}$. Although the DMA values are higher $\left(\right.$ by $\left.\sim 10^{\circ} \mathrm{C}\right)$, it can be argued that the difference does not impact freeze drying as the $\mathrm{Tg}^{\prime}$ values is based on the average of an interval range (so a degree of variability is inevitable particularly when using small volumes). Also, the FDM measured collapse values of liposome-sucrose values are similar to the known collapse temperature value of $-31^{\circ} \mathrm{C}$ for sucrose [15]. 
Table 3: The critical thermal parameters (Tg) of DMPC:Chol and DSPC:Chol liposomal formulations produced with or without OVA. The thermal analysis results of the formulations were compared using three techniques; freeze drying microscopy, modulated differential scanning calorimetry and dynamic mechanical analysis.

\begin{tabular}{llllll}
\hline Formulations & $\begin{array}{l}\text { LIPID Concentration } \\
(\mathrm{mg} / \mathrm{mL})\end{array}$ & Sucrose (\%) & $\begin{array}{l}\text { FREEZE } \\
\text { MICROSCOPY }\left({ }^{\circ} \mathrm{C}\right)\end{array}$ & $\begin{array}{l}\text { DRIED } \\
\text { MDSC }\left({ }^{\circ} \mathrm{C}\right)\end{array}$ & $\begin{array}{l}\text { DMA results (Tan Delta } \\
\left.\left({ }^{\circ} \mathrm{C}\right)\right)\end{array}$ \\
\hline DSPC:Chol + OVA & 4 & 7.5 & -34 & -36.2 & -23.4 \\
DMPC:Chol & 10 & 7.5 & -33 & -36.1 & -23.3 \\
DMPC:Chol + OVA & 10 & 7.5 & -39 & -36.2 & -24.2 \\
DSPC:Chol:PS + OVA & 10 & 7.5 & -36 & -36.2 & -17.4 \\
\hline
\end{tabular}


A

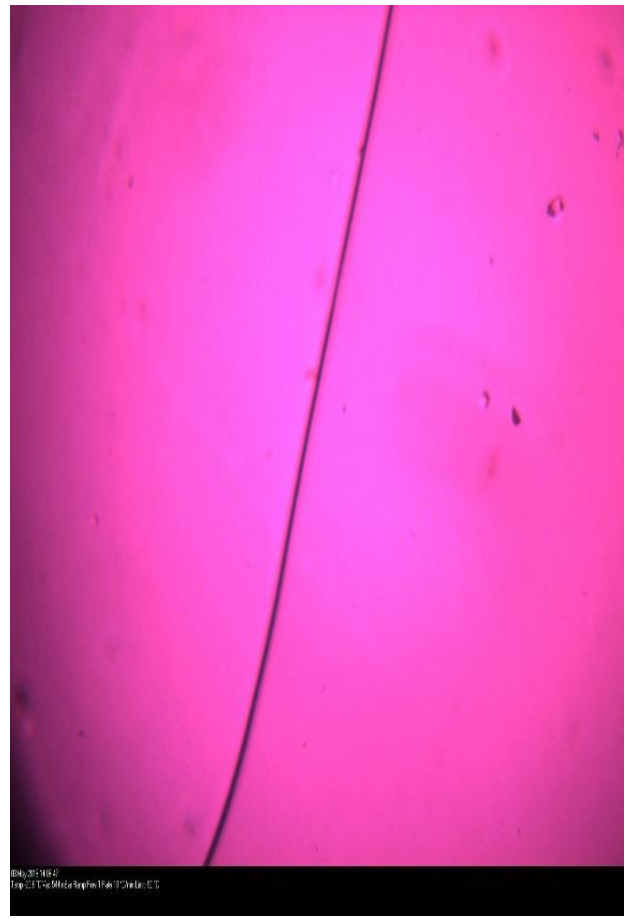

B

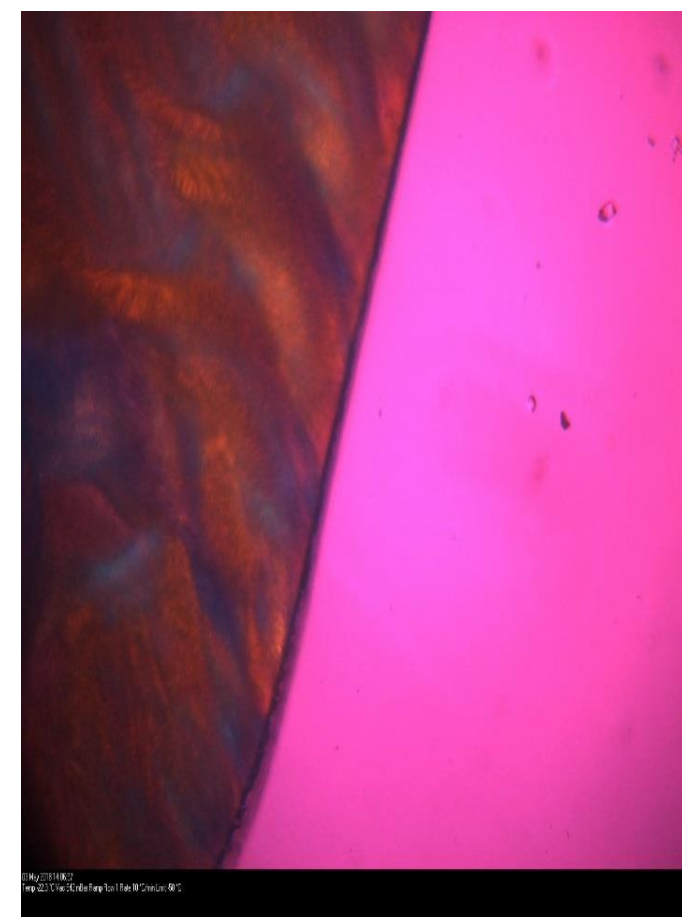

C

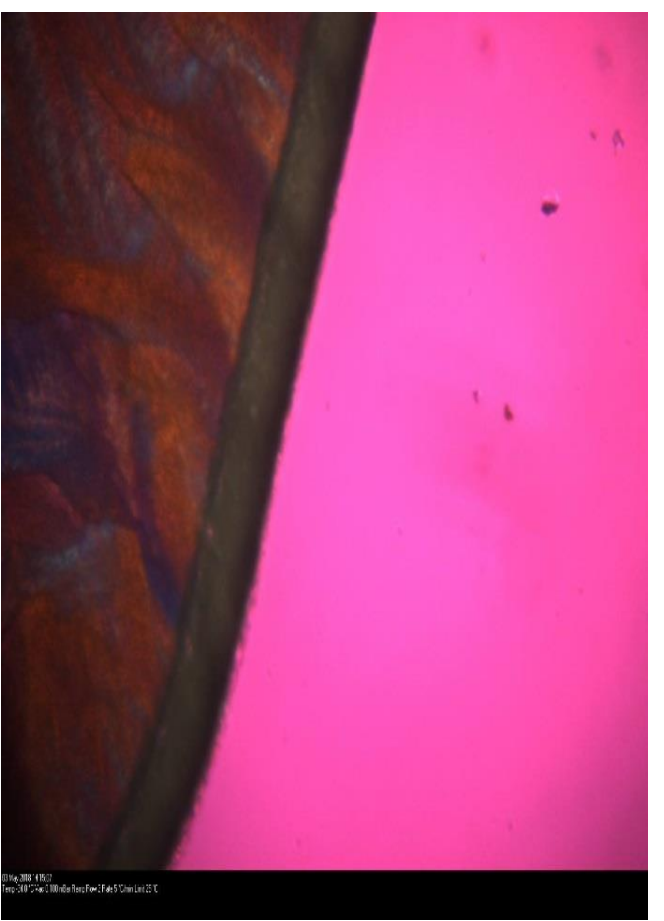

D

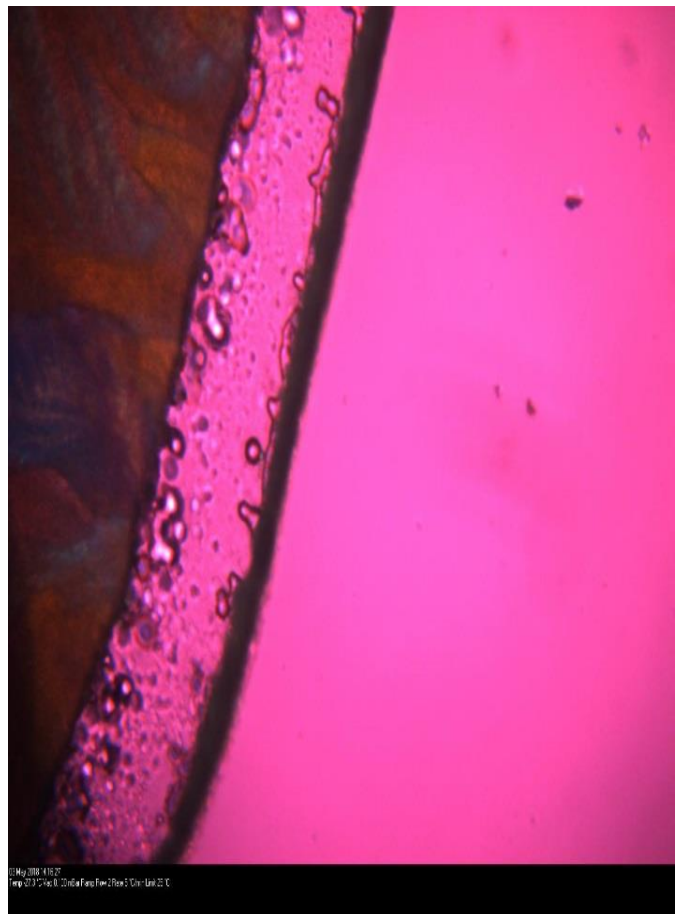

Figure 1: Freeze dried microscopy of DSPC:Chol (1 mg/mL final) with a $34 \%$ encapsulation efficiency of OVA ( $0.25 \mathrm{mg} / \mathrm{mL}$ initial OVA concentration). The formulation was added at a 1:1 v/v ratio to sucrose, producing a final concentration of $7.5 \%$ sucrose in the formulation. The sample was frozen at a rate of $10^{\circ} \mathrm{C} / \mathrm{min}$ to $-50^{\circ} \mathrm{C}(\mathrm{A})$, with the freezing point of this sample at $-18.6^{\circ} \mathrm{C}(\mathrm{B})$. This was followed by the drying phase (C) and full collapse (D) is observed at $-34^{\circ} \mathrm{C}$. 


\subsection{Characterisation of freeze dried liposomal formulations}

\subsubsection{Freeze drying of neutral liposomal formulations in either vials or microplates.}

Recent publications have highlighted the possibility of using 96 well plates to rapidly freeze dry and screen protein formulations [13]. Given the many benefits of using 96 well plates, this approach was used to investigate the freeze drying of liposomal formulations in flat bottom 96 well plates compared to vials. The 96 well plates were customised so the edges of the plates were removed to prevent edge effect. Vials and microplates were run in parallel (but on different shelves on the freeze dryer) for comparison with both formats able to form elegant cakes (Figure 2) irrespective of the lipid type (DMPC or DSPC lipid), lipid concentration, protein (presence or concentration) and, the ratio of cryoprotectant added. Results from Figure 2 , show even and reproducible cakes formed in both freeze drying formats. The geometry was key; the flat bottom of both the vials and microplates allowed for direct contact with the shelf, enabling even freeze drying to occur without the need for specialist equipment $[12,13]$ and so similar visual appearance between the vials and microplates was achievable.

Furthermore, rehydration of the cake (with water) was rapid, taking only a couple of seconds for cakes formed in microplates and vials. The use of vials or microplates did not impact the freeze drying of the liposome formulations with similar physicochemical results (size, PDI and EE) results measured for both. This trend was observed regardless of the liposomal formulation characteristics; neutral, anionic and cationic formulations were the same irrespective of the freeze drying format. For instance, neutral DMPC:Chol liposome size measured after reconstitution either in vials or microplates, remained unchanged (Figure 3A). As expected, samples freeze dried without sucrose showed a significant increase in size and PDI (results not shown). The samples freeze dried in vials show a $30-34 \%$ increase in size for empty DMPC:Chol liposomes ( 4 and $10 \mathrm{mg} / \mathrm{mL}$ ) mixed with either 5 or $10 \%$ sucrose, whilst microplates also show on average a $30 \%$ increase in liposome size post reconstitution. The effect of final cryoprotectant concentration on liposome preservation was investigated. A minimum ( $5 \%$ final) and maximum ( $10 \%$ final) sucrose concentration was selected. The concentrations were chosen with respect to previous reports that have shown disaccharide sugars are better at freeze drying products [21-23], with higher concentrations (between 5- 10\% final disaccharide at a 1:1 ratio) the most effective [21]. The results from this study (Figure 3 ) show a higher sucrose percentage (10\% final) was better for the freeze drying of liposomal formulations, in comparison to a lower percentage of sucrose (5\% final).

In contrast, OVA loaded liposomes do not increase in size as much as empty liposomes; for OVA loaded DMPC:Chol liposomes a $12-21 \%$ increase in size is observed. The PDI of all formulations remains below 0.2 after reconstitution, with no loss of OVA protein observed (Table 4). The results for the DSPC:Chol liposomes are in keeping with that observed for DMPC:Chol liposomes- no difference between vials and microplates is observed (Figure 4). For instance, empty DSPC:Chol liposomes more than double in size at both lipid concentrations ( 4 and $10 \mathrm{mg} / \mathrm{mL}$ ) from $47-56 \mathrm{~nm}$ to $113-140 \mathrm{~nm}$, whilst the increase in size for OVA loaded DSPC:Chol liposomes was not as prominent (65- $88 \mathrm{~nm}$ to $117-132 \mathrm{~nm}$ ) (Figure 4A). The PDI remained below 0.2 for all test conditions (Figure 4B).

Furthermore, the effect of freeze drying charged liposomal formulations was investigated. The results show anionic liposomal formulation DSPC:Chol:PS without the inclusion of OVA resulted in $~ 700 \%$ increase in average particle size following reconstitution. This occurred regardless of whether the freeze drying occurred in a microplate or vials (Figure 5A). However, following OVA entrapment, a 14\% 
increase in average particle size was observed following reconstitution. Conversely, cationic liposomal formulation DSPC:Chol:DOTAP showed large increases in particle size following reconstitution $(>300 \%)$ regardless of the freeze drying vessel or the inclusion of OVA, therefore further analysis of this formulation was halted for this study. Polydispersity index of all the anionic formulations tested remained below 0.25 (Figure $5 \mathrm{~B}$ ), while cationic formulations increased significantly following reconstitution $(p<0.05)$. The OVA retention of the anionic formulation remained at $98.7 \%$ following freeze drying with $367.6 \mu \mathrm{g} / \mathrm{mL}$ of protein being quantified following reconstitution.

A

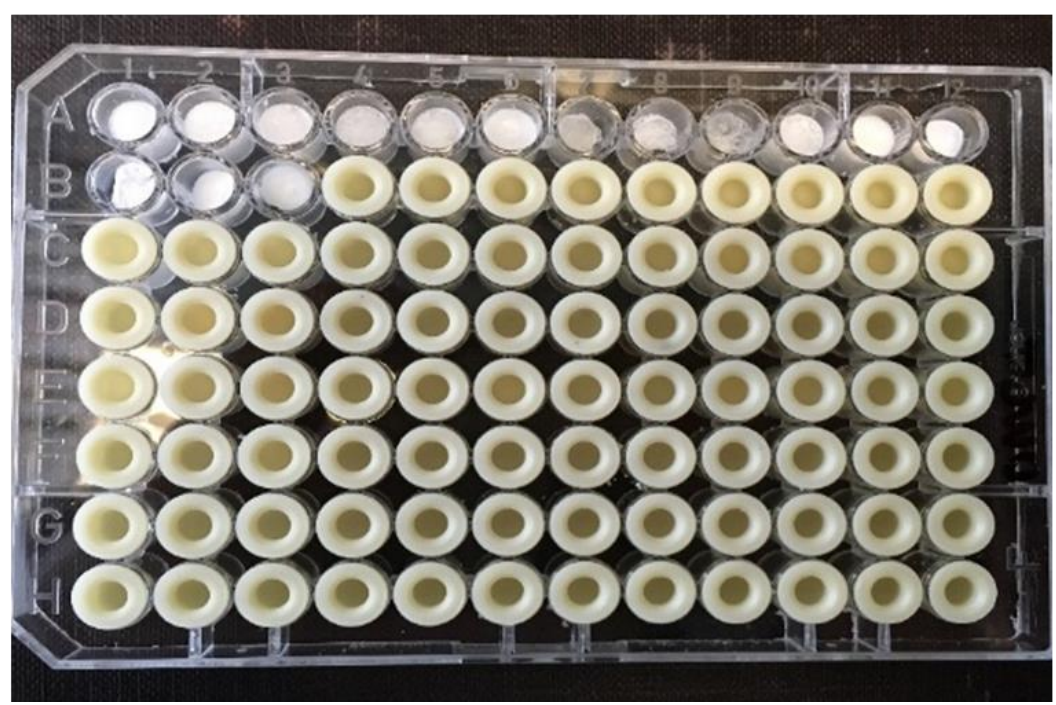

B

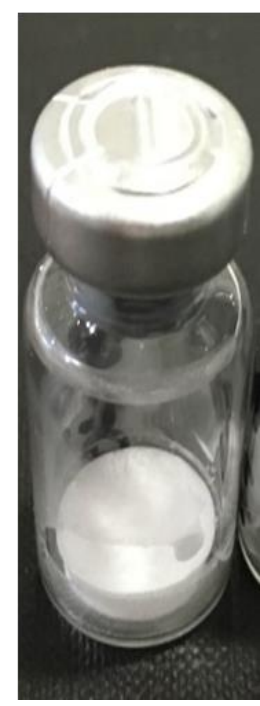

Figure 2: DMPC:Chol freeze dried in either a microplate (A) or a conventional vial (B). 


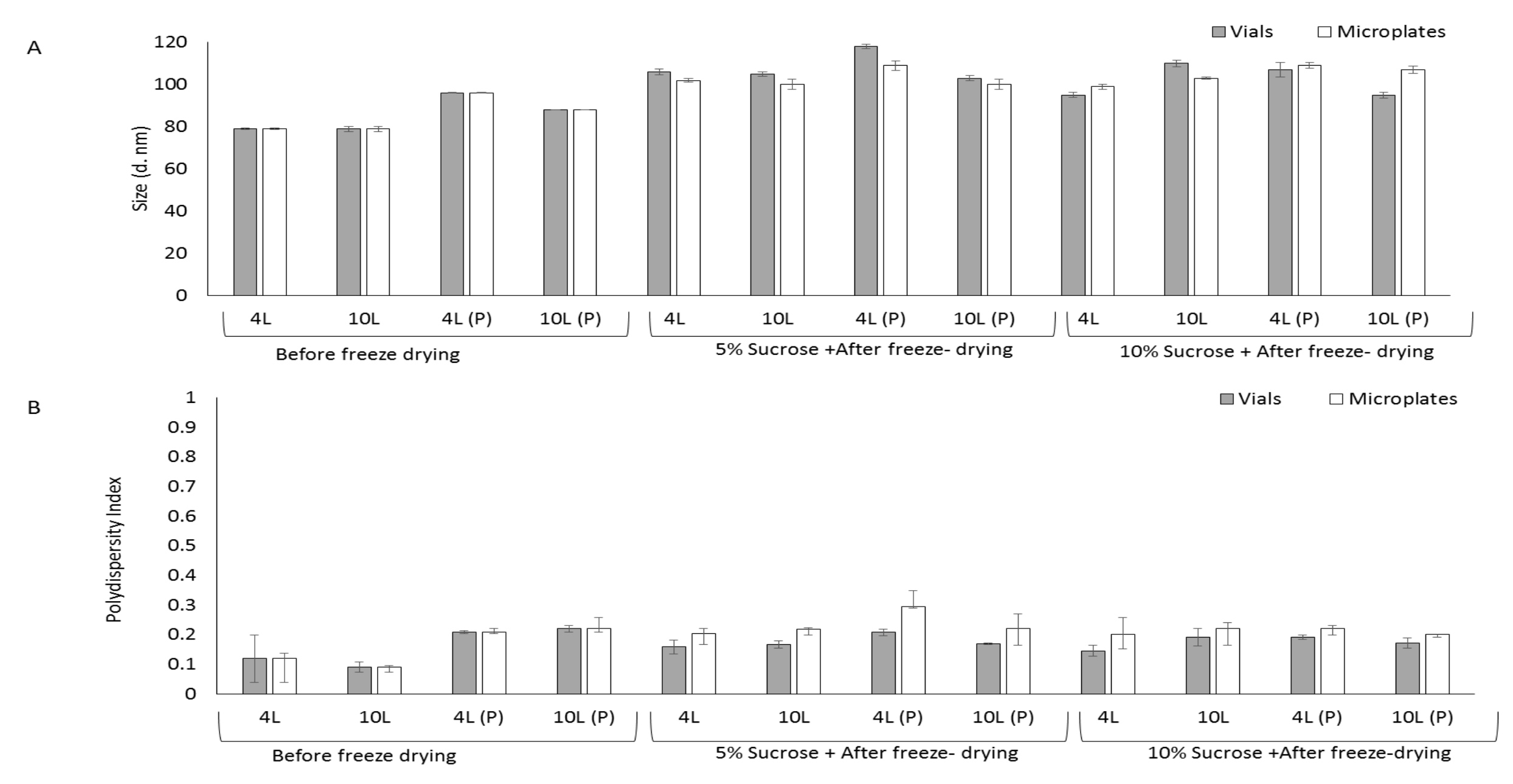

Figure 3: Characterising empty and OVA loaded $(0.25 \mathrm{mg} / \mathrm{mL})$ DMPC:Chol liposomes before and after freeze drying. The formulations were made using microfluidics at a $3: 1$ flow rate ratio and a speed of $15 \mathrm{~mL} / \mathrm{min}$, at either 4 or $10 \mathrm{mg} / \mathrm{mL}$ initial lipid concentration (represented by ' $\mathrm{L}^{\prime}$ ). The OVA encapsulation efficiency (\%) of DMPC:Chol liposomes $(0.25 \mathrm{mg} / \mathrm{mL}$ initial OVA) are represented by the letter ' $\mathrm{P}$ '. The liposomal formulations were mixed with either 5 or $10 \%$ final sucrose concentration (at a 1:1 liposome:cryoprotectant mixture) with the size (A) and polydispersity (B) measured. The results represent three independent batches, \pm SD. 


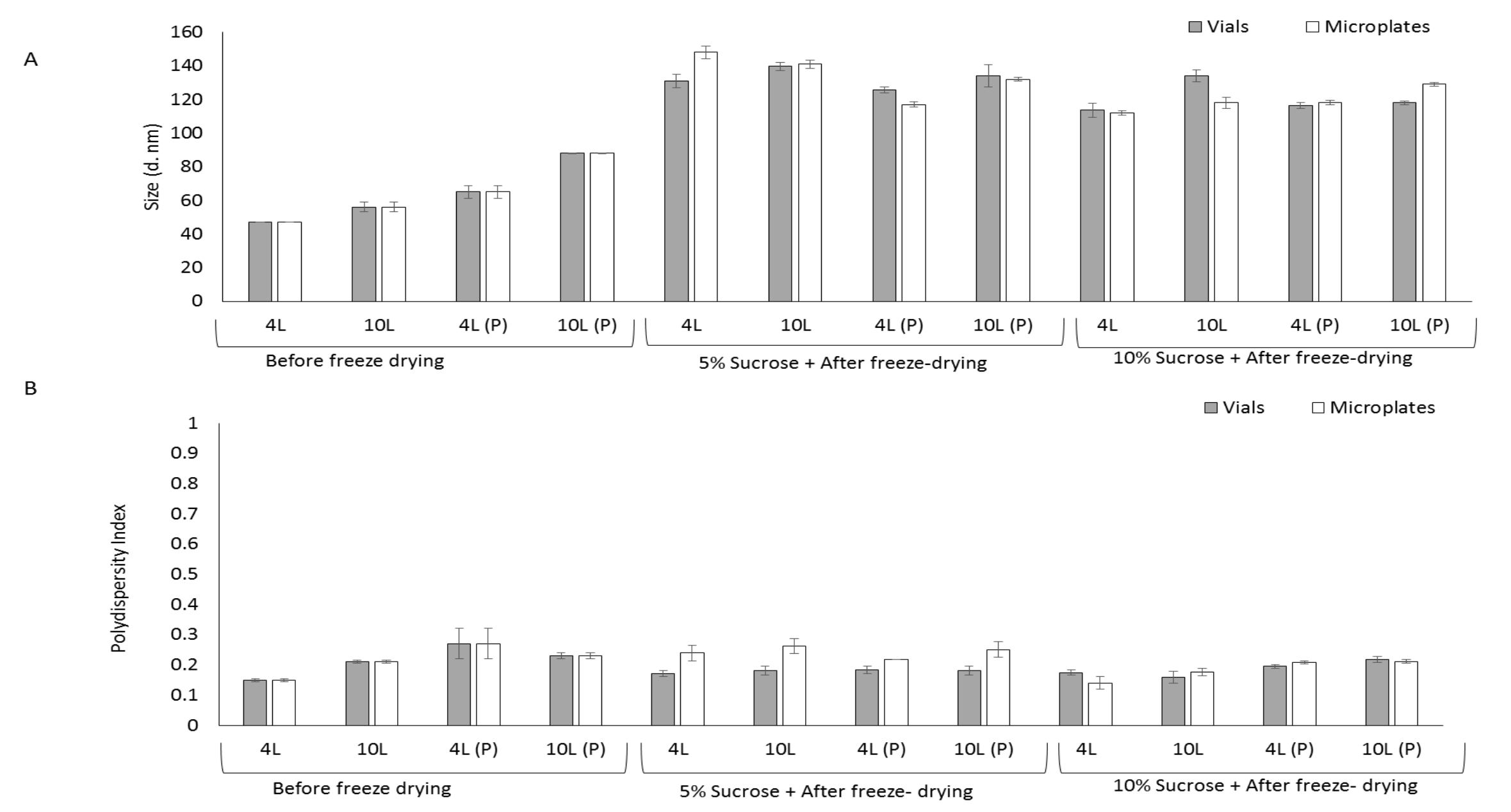

Figure 4: Characterising empty and OVA loaded $(0.25 \mathrm{mg} / \mathrm{mL})$ DSPC:Chol liposomes before and after freeze drying. The formulations were made using microfluidics at a 3:1 flow rate ratio and a speed of $15 \mathrm{~mL} / \mathrm{min}$, at either 4 or $10 \mathrm{mg} / \mathrm{mL}$ (represented by L). The DSPC:Chol liposomes containing OVA are represented by the letter ' $\mathrm{P}$ '. The liposomal formulations were mixed with either 5 or 10\% final sucrose concentration (at a 1:1 liposome:cryoprotectant mixture) with the size (A) and polydispersity (B) measured. The results represent three independent batches, \pm SD. 


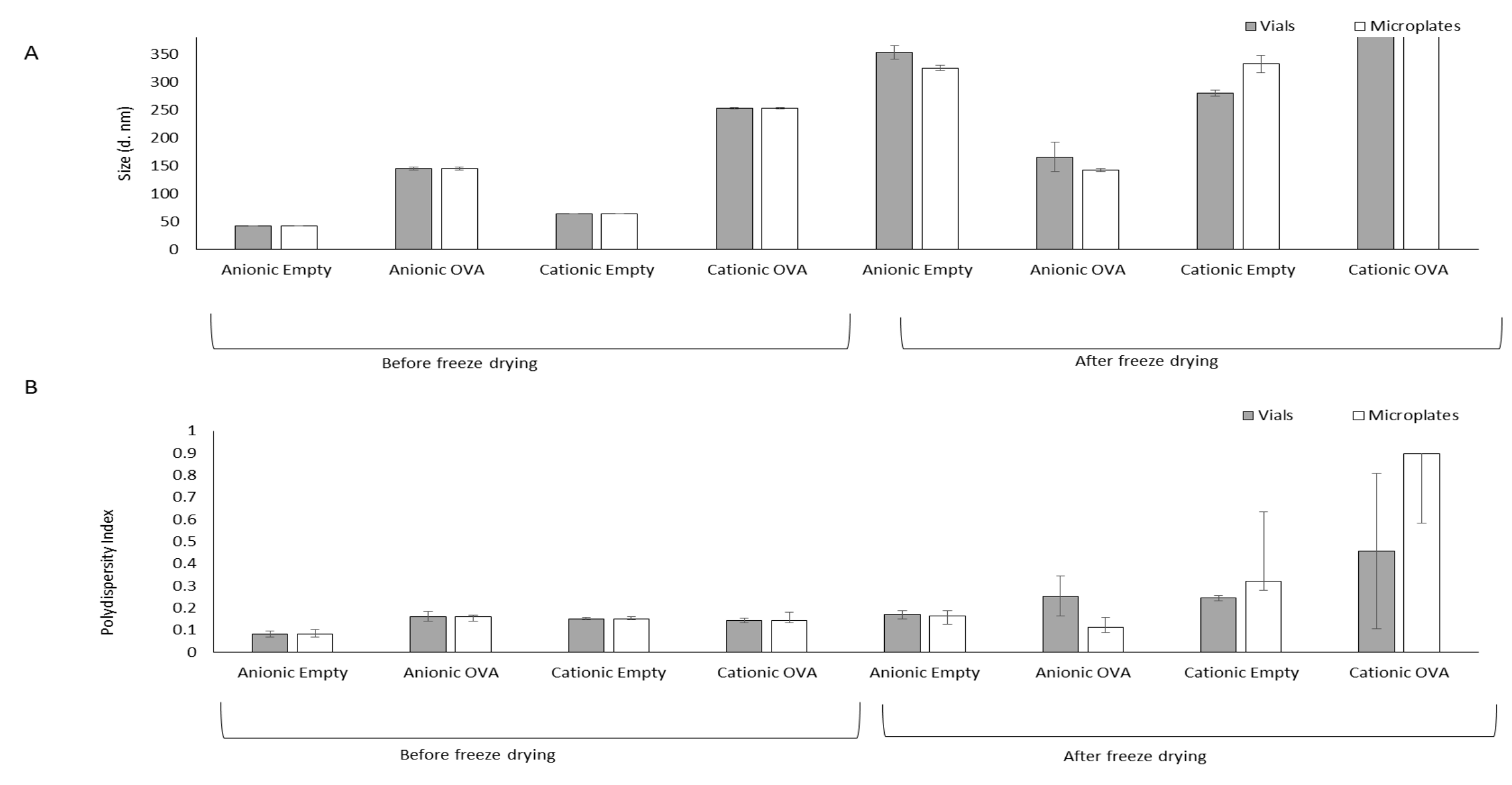

Figure 5: Characterising empty and OVA loaded DSPC:Chol:PS (anionic) and DSPC:Chol:DOTAP (cationic) liposomes before and after freeze drying. The formulations were made using microfluidics at a 3:1 (anionic) and a 1:1 (cationic) flow rate ratio and a speed of $15 \mathrm{~mL} / \mathrm{min}$, at $10 \mathrm{mg} / \mathrm{mL}$ initial total lipid. The liposomal formulations were mixed with 7.5\% final sucrose concentration (at a 1:1 liposome:cryoprotectant mixture) with the size (A) and polydispersity (B) measured. 
Table 4: Characterising empty and OVA encapsulation efficiency (as a \% of $0.25 \mathrm{mg} / \mathrm{mL}$ initial OVA) DMPC:Chol and DSPC:Chol liposomes post freeze-drying. A range of formulation variables including lipid concentration, type and amount of final sucrose concentration was investigated. The formulations were freeze dried using freeze-drying cycle 1 parameters, and rehydrated with water. The physicochemical properties (size and polydispersity) was measured using dynamic light scattering, with encapsulation calculated using HPLC-ELSD. The results represent three batches, \pm SD.

\begin{tabular}{|c|c|c|c|c|c|}
\hline FORMULATIONS & Lipid concentration $(\mathrm{mg} / \mathrm{mL})$ & Ovalbumin (mg/mL) & Sucrose (\%) & EE Before (\%) & EE After (\%) \\
\hline & 4 & 0.25 & 5 & $37 \pm 0.2$ & $36 \pm 0.2$ \\
\hline & 4 & 0.25 & 10 & $37 \pm 0.2$ & $37 \pm 0.3$ \\
\hline \multicolumn{6}{|l|}{ DMPC:Chol } \\
\hline & 10 & 0.25 & 5 & $39 \pm 0.3$ & $33 \pm 1.0$ \\
\hline & 10 & 0.25 & 10 & $39 \pm 0.3$ & $40 \pm 3.0$ \\
\hline & 4 & 0.25 & 5 & $34 \pm 0.3$ & $30 \pm 0.7$ \\
\hline & 4 & 0.25 & 10 & $34 \pm 0.3$ & $33 \pm 0.2$ \\
\hline \multicolumn{6}{|l|}{ DSPC:Chol } \\
\hline & 10 & 0.25 & 5 & $36 \pm 0.5$ & $35 \pm 1.1$ \\
\hline & 10 & 0.25 & 10 & $36 \pm 0.5$ & $34 \pm 0.4$ \\
\hline
\end{tabular}




\subsubsection{Design of experiments to identify the optimal freeze drying conditions for liposomal formulations}

The results obtained for the neutral liposomal formulations post- rehydration were then fed-back into the DoE software for both DMPC:Chol and DSPC:Chol liposomes. A full factorial was designed to investigate the effect of lipid concentration, presence of protein and sucrose concentration as these factors are known to impact the physicochemical properties (size, polydispersity index and encapsulation efficiency) of liposomes (see Table 1 for list of formulations tested). The DMPC:Chol and DSPC:Chol formulations were investigated independently (with 11 parameters for each formulation). The ideal outcome for the freeze drying of the formulations was minimal changes in size, PDI and encapsulation efficiency.

Considering the size as the variable outcome, the results show a higher starting lipid and sucrose concentration, and the presence of protein minimises the changes in physicochemical properties after freeze drying reconstitution. The presence of OVA helped stabilise the formulation, but the protein concentration did not make a difference. The minimal effect of the protein concentration on encapsulation is in keeping with previous work by Forbes et al, where it was shown the encapsulation efficiency is largely effected by the manufacturing process (in this case microfluidics) rather than the formulation composition ${ }^{17}$. Based on the results, the ideal parameters identified by DoE was $10 \mathrm{mg} / \mathrm{mL}$ lipid, $0.25 \mathrm{mg} / \mathrm{mL}$ OVA with the addition of $10 \%$ sucrose (final concentration at a $1: 1 \mathrm{v} / \mathrm{v}$ liposome:sucrose) for both formulations. Despite these favourable formulation parameters, an increase in liposomal size was observed post rehydration (Figure 4 and 5). It was speculated that the increase in size may be due to the freeze drying cycle, in particular the freezing portion of the cycle. Previous studies have shown getting the freezing process and temperature is particularly important when freeze drying liposomes as they are extremely sensitive to changes in temperature and the formation of ice crystals [24-26]. As a result, these parameters were used for further freeze drying of liposome formulations.

\subsubsection{Optimising and validating the freeze dried cycle for protein loaded liposome formulations}

The results from FDC1 method resulted in an increase in liposomal size post- rehydration (section 3.3.2). Due to this, using the best formulation parameters determined using DoE, two alternative freeze drying cycles were investigated (FDC2 and FDC3). The formulation parameters used included a $10 \mathrm{mg} / \mathrm{mL}$ initial lipid concentration and with an OVA encapsulation efficiency of $36 \%(0.25 \mathrm{mg} / \mathrm{mL}$ initial OVA concentration). The liposomes-sucrose mixture had a final concentration of $10 \%(\mathrm{v} / \mathrm{v})$, with the samples placed in vials before undergoing freeze drying.

The two freeze drying cycle adaptations focused on changes to the freezing techniques. The samples were either ramp frozen (FDC2) or snap frozen using liquid nitrogen (FDC3), to investigate if this change is able to maintain liposome characteristics (Table 5). Both freezing techniques resulted in elegant cakes (pictures not shown). While both approaches produced a homogenous population post rehydration of freeze dried samples (Figure 6), an increase of $15 \mathrm{~nm}$ (from $85 \pm 0.8 \mathrm{~nm}$ to $100 \pm 0.3 \mathrm{~nm}$ ) for DSPC:Chol liposomes size occurred, whilst no change in size occurred ( $85 \pm 0.4 \mathrm{~nm}$ ) for DMPC:Chol liposomes (Figure 6). The small increase in size may be due to smaller liposomes being more susceptible to changes to freeze drying as they have a larger surface area and unstable [27]. Rehydration may cause the water to enter the aqueous core, causing swelling and thus an increase in liposome size [28]. 
Table 5: Determining the amount of OVA retained after freeze- drying OVA loaded DMPC:Chol and DSPC:Chol liposomes, with either 5 or $10 \%$ final sucrose concentration (1:1 (v/v) liposomes to cryoprotectant). The liposomal formulations were freeze dried using either freeze-dried cycle 2 or freeze-dried cycle 3. Post rehydration the amount of encapsulated OVA was calculated using HPLC-ELSD to determine if any leakage of OVA had occurred. The results represent three batches, mean \pm SD.

\begin{tabular}{|c|c|c|c|c|c|}
\hline FORMULATIONS & Lipid concentration $(\mathrm{mg} / \mathrm{mL})$ & Ovalbumin (mg/mL) & Sucrose (\%) & EE Before (\%) & EE After (\%) \\
\hline \multirow{5}{*}{ DMPC:Chol } & 4 & 0.25 & 5 & $37 \pm 0.2$ & $36 \pm 0.2$ \\
\hline & 4 & 0.25 & 10 & $37 \pm 0.2$ & $37 \pm 0.3$ \\
\hline & & & & & \\
\hline & 10 & 0.25 & 5 & $39 \pm 0.3$ & $33 \pm 1.0$ \\
\hline & 10 & 0.25 & 10 & $39 \pm 0.3$ & $40 \pm 3.0$ \\
\hline \multirow{5}{*}{ DSPC:Chol } & 4 & 0.25 & 5 & $34 \pm 0.3$ & $30 \pm 0.7$ \\
\hline & 4 & 0.25 & 10 & $34 \pm 0.3$ & $33 \pm 0.2$ \\
\hline & & & & & \\
\hline & 10 & 0.25 & 5 & $36 \pm 0.5$ & $35 \pm 1.1$ \\
\hline & 10 & 0.25 & 10 & $36 \pm 0.5$ & $34 \pm 0.4$ \\
\hline
\end{tabular}




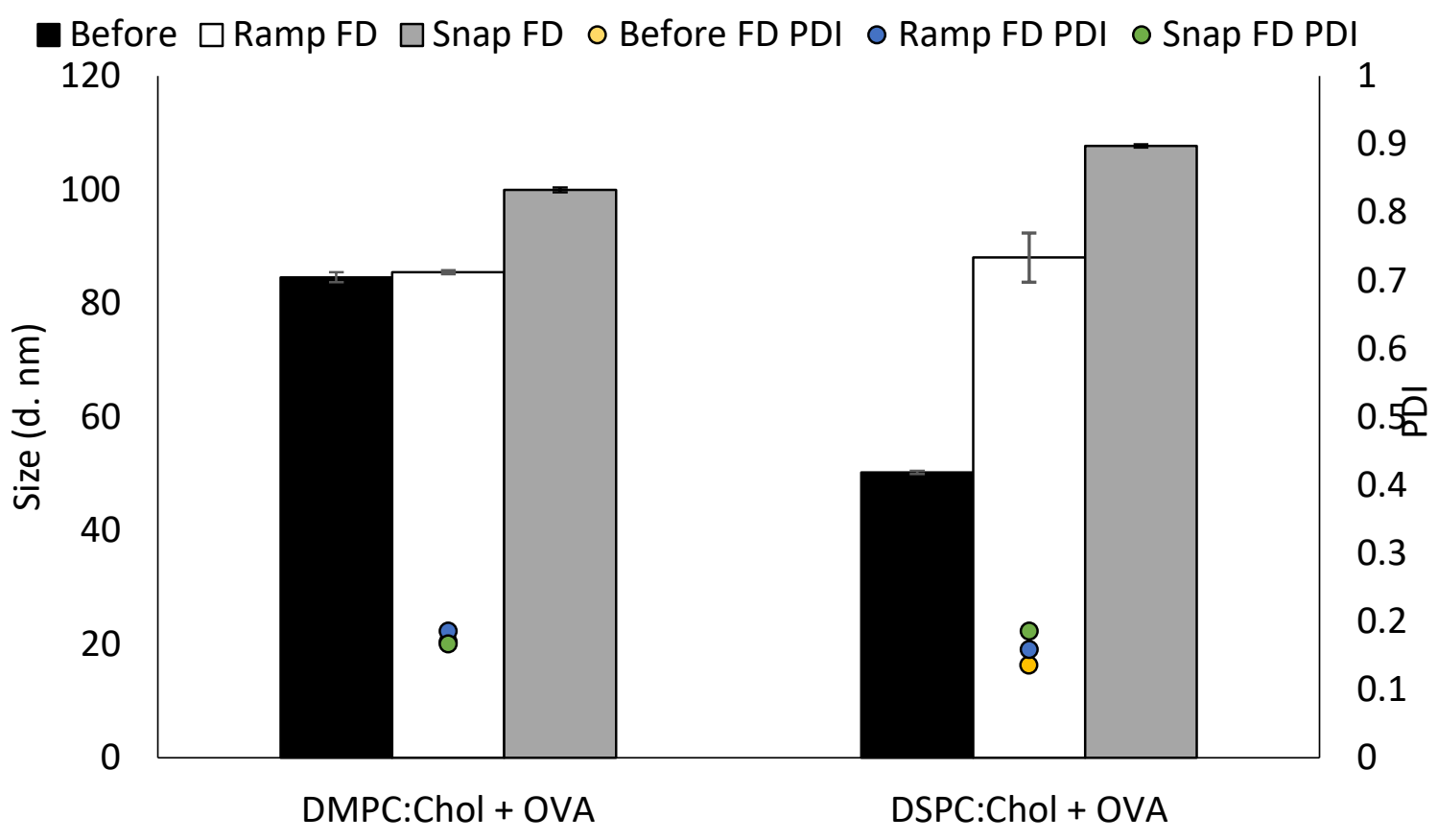

Figure 6: The characterisation of OVA loaded DMPC:Chol and DSPC:Chol liposomes before and after freeze drying. The liposomes either underwent ramp freezing or snap freezing as part of the freeze drying cycle. All samples were freeze dried in the presence of the cryoprotectant, sucrose $(10 \% \mathrm{v} / \mathrm{v}$ at a 1:1 ratio). The results represent three independent batches, \pm SD.

\subsubsection{Residual moisture content results}

The residual moisture content analysis has shown differences in residual moisture depending on the freeze dried approach used. Ramp freezing produces formulations with a residual moisture content of below $2 \%$ whilst the measured moisture content for snap frozen formulations is above $2 \%$. The results indicate extension of the primary drying phase for 22 hours for FDC2 (compared to 10 hours for FDC3) has a beneficial effect on the residual moisture content (Table 6). Little difference between the two formulations (DMPC:Chol and DSPC:Chol) was observed, with the freeze drying cycle largely influencing the amount of residual moisture content. The residual moisture content for ramp frozen DMPC:Chol and DSPC:Chol was 1.0 and $1.35 \%$ respectively, compared to $2.30 \%$ and $2.44 \%$ for snap frozen methods.

Furthermore, the residual moisture results show that the FDC2 method is better for freeze drying liposomal formulations as it is able to achieve a residual moisture below $2 \%$. This is important as previous literature has shown a residual moisture of greater than $2 \%$ can impact the stability of the product in the long run [29]. Whilst a moisture content below a particular level is not stipulated, the International Conference on Harmonisation (ICH) Q8 (R2) has provided examples whereby freezedried liposomal formulations with a residual moisture content of less than $2 \%$ have been produced (ICH Q8 (R2)[30]). 
Table 6: The residual moisture content of DMPC:Chol and DSPC:Chol liposomes freeze dried under ramp or snap frozen conditions, containing the lyoprotectant sucrose. The results represent two batches, each the mean of two determinations.

\begin{tabular}{lll}
\hline Formulation & Moisture $\mathbf{w} / \mathbf{w} \%$ & \\
\hline & Ramp frozen (\%) & Snap frozen (\%) \\
DMPC & 1.0 & 2.30 \\
DSPC & 1.35 & 2.44 \\
$20 \%$ sucrose & 1.55 & - \\
\hline
\end{tabular}

\section{Discussion}

In this research study, the effect of the formulation parameters as well as freeze drying cycle conditions on liposomal preservation was investigated. The formulation parameters chosen were based on previous literature research which had shown the presence of protein, cholesterol, the lipid charge, concentration and cryoprotectant concentration can influence liposome stability. As a result, some of these formulation parameters were tested using a DoE approach. The formulations were freeze-dried under the first freeze drying cycle developed using analytical techniques.

The first freeze-drying cycle was developed using the collapse temperature and $\mathrm{Tg}^{\prime}$ values from FDM and MDSC respectively. The collapse temperature for all formulations tested was between -39 and $33^{\circ} \mathrm{C}$, with a $\mathrm{Tg}^{\prime}$ of $-34^{\circ} \mathrm{C}$ determined by MDSC. Based on these results, the samples were frozen at $45^{\circ} \mathrm{C}$ with primary drying held at $-30^{\circ} \mathrm{C}$ (slightly higher than the $\mathrm{Tg}^{\prime}$ value determined). This was done due to numerous research showing a lag between sample temperatures compared to the shelf temperature during the primary drying phase. The secondary drying phase was selected to be above $0^{\circ} \mathrm{C}$ to ensure maximum removal of any residual moisture. Briefly, post- rehydration of the DoE formulations identified certain parameters had a greater impact on formulation stability. The presence of protein adds stability to both neutral and charged formulations, which is in keeping with previous literature [31] with the same amount of OVA retained after freeze drying (Table 4). The minimal to no leakage of the OVA suggest that pre-cooling the shelf and freezing is ideal at preventing leakage- it caused the formation of large crystals [26]. In contrast, the liposomal size changed upon rehydration, with cationic liposomes showing the greatest increase in size. These observations were the same regardless of whether the samples underwent freeze drying using vials or 96 well plates. Based on the sample results, and the analysis of pressure rise test traces (results not shown) it was concluded the whole batch has not undergone complete sublimation. Changes to the freeze drying cycle were made, with two alternative cycles developed to determine if this could maintain liposomal physicochemical properties.

Furthermore, the freeze drying conditions were adjusted and two different freezing approaches (ramp freezing (FDC2) and snap freezing (FDC3)), in addition to changes to the primary and secondary drying were evaluated. The results show the snap freeze method (FDC3) caused an significant increase in size for DMPC:Chol and DSPC:Chol liposomes $(p<0.001)$. Meanwhile, the ramp frozen method caused 
minimal changes in liposomal physicochemical properties. Both freeze drying cycles were able to retain more than $99 \%$ of encapsulated OVA (Table 5). In corroboration, the residual moisture content of below $2 \%$ for ramp frozen formulations indicate the production of stable formulations, with minimal leakage and preserved characteristics. Therefore, the results show a successful method for the rapid freeze drying of liposomal formulations using the ramp freezing approach.

\section{Conclusion}

The parameters and processes needed for the rapid freeze drying of liposomal formulations containing enclosed protein were investigated. We show the ability to freeze dry liposomal formulations in microplates as well as vials, for the rapid screening, preservation and optimization of liposomal formulations. Liposomal physicochemical characteristics were preserved irrespective of the formulation type, with no loss of protein observed. Whilst the freeze-drying cycle was effective for PC derivative formulations, future work will be needed to investigate a wider range of liposomes. Nonetheless, the freeze drying process developed offers a feasible, transferable and rapid quantifiable method for screening and improving the longevity potential of pharmaceutical liposomal products.

\section{Acknowledgments}

This work was funded by the University of Strathclyde (Glasgow, UK) and by the EPSRC Centre for Innovative Manufacturing in Emergent Macromolecular Therapies (EP/L015218/1). 


\section{References}

1. Fenske, D.B. and P.R. Cullis, Liposomal nanomedicines. Expert opinion on drug delivery, 2008. 5(1): p. 25-44.

2. Torchilin, V.P., Recent advances with liposomes as pharmaceutical carriers. Nature reviews Drug discovery, 2005. 4(2): p. 145.

3. Zuidam, N.J., et al., Physical (in) stability of liposomes upon chemical hydrolysis: the role of lysophospholipids and fatty acids. Biochimica et Biophysica Acta (BBA)-Biomembranes, 1995. 1240(1): p. 101-110.

4. Grit, M. and D.J. Crommelin, Chemical stability of liposomes: implications for their physical stability. Chemistry and physics of lipids, 1993. 64(1-3): p. 3-18.

5. Immordino, M.L., F. Dosio, and L. Cattel, Stealth liposomes: review of the basic science, rationale, and clinical applications, existing and potential. International journal of nanomedicine, 2006. 1(3): p. 297.

6. Fransen, G.J., P.J.M. Salemink, and D.J.A. Crommelin, Critical parameters in freezing of liposomes. International Journal of Pharmaceutics, 1986. 33(1): p. 27-35.

7. Meunier, F., H. Prentice, and O. Ringden, Liposomal amphotericin B (AmBisome): safety data from a phase II/III clinical trial. Journal of Antimicrobial Chemotherapy, 1991. 28(suppl B): p. 83-91.

8. Chang, H.-I. and M.-K. Yeh, Clinical development of liposome-based drugs: formulation, characterization, and therapeutic efficacy. International Journal of Nanomedicine, 2012. 7: p. 49-60.

9. Park, J.W., Liposome-based drug delivery in breast cancer treatment. Breast Cancer Research, 2002. 4(3): p. 95.

10. Hoarau, D., et al., Novel long-circulating lipid nanocapsules. Pharmaceutical research, 2004. 21(10): p. 1783-1789.

11. Chen, C., et al., An overview of liposome lyophilization and its future potential. Journal of Controlled Release, 2010. 142(3): p. 299-311.

12. Grant, Y., P. Dalby, and P. Matejtschuk, Use of design of experiment and microscale down strategies in formulation and cycle development for lyophilization. American Pharmaceutical Review, 2012. 15(2).

13. Grant, Y., P. Matejtschuk, and P.A. Dalby, Rapid optimization of protein freeze-drying formulations using ultra scale-down and factorial design of experiment in microplates. Biotechnology and bioengineering, 2009. 104(5): p. 957-964.

14. Van Winden, E. and D. Crommelin, Long term stability of freeze-dried, lyoprotected doxorubicin liposomes. European journal of pharmaceutics and biopharmaceutics, 1997. 43(3): p. 295-307.

15. Crowe, L.M., et al., Prevention of fusion and leakage in freeze-dried liposomes by carbohydrates. Biochimica et Biophysica Acta (BBA) - Biomembranes, 1986. 861: p. 131-140.

16. Gearing, J., K.P. Malik, and P. Matejtschuk, Use of dynamic mechanical analysis (DMA) to determine critical transition temperatures in frozen biomaterials intended for lyophilization. Cryobiology, 2010. 61(1): p. 27-32.

17. Forbes, N., et al., Rapid and scale-independent microfluidic manufacture of liposomes entrapping protein incorporating in-line purification and at-line size monitoring. International Journal of Pharmaceutics, 2019. 556: p. 68-81.

18. Crowe, L.M., J.H. Crowe, and D. Chapman, Interaction of carbohydrates with dry dipalmitoylphosphatidylcholine. Archives of biochemistry and biophysics, 1985. 236(1): p. 289-296.

19. Ohtake, S., et al., Phase behavior of freeze-dried phospholipid-cholesterol mixtures stabilized with trehalose. Biochimica et Biophysica Acta (BBA)-Biomembranes, 2005. 1713(1): p. 57-64. 
20. Matejtschuk, P., et al., Use of thermogravimetric analysis for moisture determination in difficult lyophilized biological samples. American Journal of Analytical Chemistry, 2016. 7(03): p. 260.

21. Crowe, J.H., et al., Stabilization of dry membranes by mixtures of hydroxyethyl starch and glucose: the role of vitrification. Cryobiology, 1997. 35(1): p. 20-30.

22. Anchordoguy, T.J., et al., Modes of interaction of cryoprotectants with membrane phospholipids during freezing. Cryobiology, 1987. 24(4): p. 324-331.

23. Hua, Z.-Z., et al., Freeze-drying of liposomes with cryoprotectants and its effect on retention rate of encapsulated ftorafur and vitamin A. Drying Technology, 2003. 21(8): p. 1491-1505.

24. Ingvarsson, P.T., et al., Stabilization of liposomes during drying. Expert opinion on drug delivery, 2011. 8(3): p. 375-388.

25. Bedu-Addo, F.K., Understanding lyophilization formulation development. Pharmaceutical Technology, 2004. 20: p. 10-19.

26. van Winden, E.C., W. Zhang, and D.J. Crommelin, Effect of freezing rate on the stability of liposomes during freeze-drying and rehydration. Pharmaceutical research, 1997. 14(9): p. 1151-1160.

27. Hsu, C.C., et al., Surface denaturation at solid-void interface-a possible pathway by which opalescent participates form during the storage of lyophilized tissue-type plasminogen activator at high temperatures. Pharmaceutical research, 1995. 12(1): p. 69-77.

28. Crowe, J.H. and L.M. Crowe, Factors affecting the stability of dry liposomes. Biochimica et Biophysica Acta (BBA) - Biomembranes, 1988. 939(2): p. 327-334.

29. Nagase, H., H. Ueda, and M. Nakagaki, Effect of water on lamellar structure of DPPC/sugar systems. Biochimica et Biophysica Acta (BBA)-Biomembranes, 1997. 1328(2): p. 197-206.

30. Guideline, I.H.T. Validation of analytical procedures: text and methodology Q2 (R1). in International Conference on Harmonization, Geneva, Switzerland. 2005.

31. Ntimenou, V., et al., Stability of protein-encapsulating DRV liposomes after freeze-drying: a study with BSA and t-PA. Journal of liposome research, 2006. 16(4): p. 403-416. 